\title{
Aproximación a la semántica del contrafactual*
}

\author{
Approximation to the semantics of counterfactuals
}

\author{
Mabel Alejandra Urrutia Martínez¹, Manuel de Vega Rodríguez² \\ ${ }^{1}$ Universidad de La Laguna, Facultad de Psicología, Departamento de Psicología Cognitiva, \\ Social y Organizacional, Tenerife, España. Correo electrónico: murrutia@ull.es \\ 2 Universidad de La Laguna, Facultad de Psicología, Departamento de Psicología Cognitiva, \\ Social y Organizacional, Tenerife, España. Correo electrónico: mdevega@ull.es
}

En este trabajo se explican aspectos fundamentales del contrafactual, como sus funciones en el razonamiento causal, en la argumentación científica, en la modulación y amplificación de las emociones. También se describen los diversos tipos de contrafactuales y las diferencias individuales en la producción y comprensión de éstos. Posteriormente, se explican las principales teorías e investigaciones que han planteado el tema del significado dual del contrafactual: los modelos mentales, las teorías proposicionales del razonamiento o la teoría suposicional. Finalmente, se dedica especial atención a las pocas investigaciones que se han realizado en el campo de la psicolingüística sobre la comprensión de contrafactuales.

Palabras clave: contrafactuales, comprensión del lenguaje, modelos mentales, teorías proposicionales, teoría de la mente.

The current study explores some fundamental aspects of counterfactuals, such us their functions in causal reasoning, scientific argument, and modulation and amplification of emotions. This study also describes the various types of counterfactuals, and the individual differences in their production and comprehension. Subsequently, the main theories and researches related to the dual meaning of counterfactuals were carried out, for example, mental models, propositional reasoning theories, or suppositional theory. Finally, special attention was given to the few investigations that have been made in the field of psycholinguistics, concerned with the comprehension of counterfactuals.

Key words: counterfactuals, language comprehension, mental models, propositional theories, theory of mind.

\section{FUNCIONALIDAD DEL CONTRAFACTUAL}

Cotidianamente se hace uso de la contrafactualidad tanto en el pensamiento como en el lenguaje. Expresiones como "si hubiera llamado a mi novio habríamos ido al

Este trabajo fue financiado por el Ministerio de Investigación e Innovación de España, mediante el proyecto de Investigación SEJ2007-66916, el proyecto Neurocog de la Agencia Canaria ACIISI y el proyecto europeo FEDER concedido a Manuel de Vega y una beca FPI (Formación Profesional Investigador) SEJ2004-02360 a Mabel Urrutia. 
cine”, "Si hubiera aparcado más cerca, habría llegado a tiempo a la cita”, "Si hubiera ganado el Girona en lugar del Tenerife...", "Si me hubiera tomado un té en lugar de un café...", "Si hubiera tenido dinero...", hacen referencia a eventos que contradicen los hechos y van unidas la mayoría de las veces a un sentimiento de pesar o lamento.

La cotidianeidad del contrafactual como expresión de pesar contrasta con la aparatosidad de su formato lingüístico, esto es, pretérito pluscuamperfecto o condicional compuesto del modo subjuntivo. El modo subjuntivo es el modo de la irrealidad por excelencia y, en el caso del contrafactual, sitúa el centro deíctico entre la realidad del hablante (su aquí y ahora) y un tiempo pasado en que transcurriría la supuesta acción. Las personas más dadas a rumiar las experiencias pasadas, especialmente con resultados negativos (v.g., adultos de avanzada edad o personas depresivas), incrementan la frecuencia de expresiones contrafactuales. Bajo el paraguas del "Si hubiera tenido tiempo..." muchos evalúan las alternativas disponibles y las posibles acciones que hubieran llevado a cabo si las condiciones hubieran sido diferentes.

Varios estudios en el ámbito de la psicología social establecen que los contrafactuales pueden ser una expresión de pesar por la acción (desear no haber hecho algo) o de pesar por la inacción (desear haber hecho algo). En el corto plazo (días o semanas) la gente suele utilizar contrafactuales de pesar por la acción (tendría que haberme callado), ya que las acciones inmediatas tienen un gran impacto en nosotros. Por el contrario, en el largo plazo (meses o años) se utilizan más contrafactuales de inacción (debería haber dedicado más tiempo a mis hijos) ya que los eventos, lejanos en el tiempo, no implican riesgos en el momento actual y sólo están limitados por nuestra imaginación (Kahneman y Tversky 1982; Gilovich y Medvec 1994).

Ahora bien, los contrafactuales no son una mera recreación retrospectiva de lo que no ocurrió en el pasado, sino la iniciación de un proceso constructivo orientado al futuro a través de la simulación mental de nuevas posibilidades válidas en un futuro inmediato (Roese et al. 2005). Bajo el parámetro de las oportunidades, los contrafactuales ayudan a prevenir errores, proporcionando beneficios a corto plazo, especialmente cuando el pensamiento contrafactual está asociado a eventos recurrentes o de alta frecuencia que probablemente tendrán oportunidad de repetirse. Sin embargo, a largo plazo, el pensamiento contrafactual obsesivo sobre los mismos hechos del pasado tiene una funcionalidad psicológica dudosa. Por ejemplo, hay acciones o inacciones en el pasado remoto que han tenido unas consecuencias estratégicas en la orientación vital del individuo y cuya evocación contrafactual no resulta útil, ya que son oportunidades perdidas, por tanto no hay nada que se pueda hacer para remediarlas, ni en el presente ni en el futuro. Éste es el caso de eventos extraordinarios, esto es, sucesos que ocurren sólo una vez en la vida y de cuya elección dependen muchas nuevas decisiones. Por ejemplo, la elección de una carrera es una oportunidad que normalmente aparece sólo una vez y de esa elección depende el trabajo, el salario, las relaciones interpersonales e incluso el modelo de familia al que se puede optar. En definitiva, los contrafactuales son útiles para modificar conductas en situaciones recurrentes a corto plazo, en cuyo caso el pesar que conlleva un resultado negativo sirve de aliciente para un futuro próximo; sin embargo, el pesar que provocan las decisiones ante eventos únicos, como haber elegido mal una carrera, puede durar toda la vida y la posibilidad de corregir ese error tal vez nunca llegue a suceder (ver Markman et al. 2009). 
En todo contrafactual hay un proceso de inferencia causal subyacente, que se expresa mediante la cláusula condicional antecedente y la cláusula consecuente. Por este motivo, los contrafactuales desempeñan un papel en el razonamiento, las atribuciones causales y la toma de decisiones. Así ante un resultado negativo en una situación determinada, la simulación contrafactual de un escenario hipotético alternativo permite comparar distintas posibilidades de un mismo evento y establecer vínculos causales que podrán ser útiles para afrontar contingencias futuras similares (Roese y Olson 1995).

De acuerdo con lo anterior, se destacan dos tipos de contrafactuales: ascendente (upward) y descendente (downward). El primer tipo se establece a través de una comparación directa entre una situación real negativa y una posibilidad mejor, que hubiéramos preferido que ocurriera. Así en "Si hubiera estudiado para el examen, habría aprobado el curso”, el contrafactual sirve como una especie de instrucción para mejorar la acción futura. Distinto es el caso de los contrafactuales descendentes como "Si hubiera subido a ese avión, me habría muerto al estrellarse”, donde los eventos podrían haber sido peores (MacMullen et al. 1995).

El contraste contrafactual entre la realidad y lo que podría haber sido induce o amplifica las llamadas "emociones contrafactuales" como la frustración, el lamento, la culpa o la vergüenza, emociones típicas asociadas a los contrafactuales ascendentes, o bien los sentimientos de alivio, satisfacción, bienestar y suerte, característicos de los contrafactuales descendentes. Los contrastes entre la realidad y sus alternativas, derivadas de un contrafactual, provocan una especie de compensación entre los eventos y las emociones. Esto es, en los contrafactuales descendentes, la alternativa imaginaria es negativa y el sentimiento que lo acompaña es de consolación; en cambio, en los contrafactuales ascendentes la alternativa imaginaria es positiva y los sentimientos que provoca son negativos como el sentimiento de pesar (Roese 2005).

\section{DIFERENCIAS INDIVIDUALES EN EL USO DEL CONTRAFACTUAL}

La gente optimista tendería a generar más contrafactuales ascendentes que los pesimistas (v.g., "Si hubiera estudiado el fin de semana habría aprobado el examen de Matemáticas"), debido a que se sienten motivados hacia la acción futura que evite errores pasados, y experimentando una sensación de control (v.g.: "Con unas horas más de estudio, seguro que apruebo el examen de Matemáticas”). También es cierto que una persona optimista puede utilizar eventualmente contrafactuales descendentes, celebrando así el haberse salvado de un mal resultado, por ejemplo, un accidente de tráfico. La funcionalidad del contrafactual ascendente o descendente dependerá del tipo de situación problema, del grado en que fomenta el desarrollo de acciones apropiadas, y de la intensidad de la emoción resultante. Todo ello conduce a un balance entre coste y beneficio y al parecer, ambos se activarían equilibradamente en alguien con una personalidad optimista. En cambio, ciertos rasgos negativos de personalidad pueden determinar el uso de contrafactuales ascendentes como es el caso de las personas pesimistas que tienden a atribuir los resultados negativos a su propia actuación o incluso a su forma de ser. Así en "Si hubiera sido más inteligente, había aprobado el examen”, "Si hubiera sido más alto, habría jugado baloncesto", "Si hubiera nacido algo diferente..."; o en situaciones adversas como accidentes de 
tráfico "Si hubiera ido por otra vía, no habríamos chocado contra aquel camión", "Si me hubiera quedado en casa el día de la tragedia", etc.

En general, los seres humanos, con el afán de mantener el control, también tienden a responsabilizarse por los eventos negativos. Así en "Si hubiera preparado mejor las clases, los alumnos habrían sacado mejores notas", "Si le hubiera demostrado mi cariño, no me habría abandonado". Esto ocurre especialmente en dominios vitales relacionados con la carrera, las relaciones humanas, la salud, la familia, las finanzas, los amigos, etc., en los cuales hay un alto porcentaje de eventos que no están bajo nuestro control, pues dependen de la actuación de otros o del azar. Quizás uno de los dominios que tiene mayor posibilidad de cambio se encuentre en el terreno personal, (v.g., “¿Qué hubiera sido de mi vida si me hubiera casado con otra persona?”), a diferencia del dominio laboral en la selección de una carrera (v.g., "Si hubiera estudiado humanidades en lugar de ciencia...”), donde hay menos posibilidades para mejorar el futuro, dado que varios factores determinan la elección de una carrera y muchas veces es imposible volver atrás.

Distinto es el caso de los individuos con una conducta negligente o de autocomplacencia, aquellos que dejan todo para mañana. En estos casos, la frecuencia de uso de contrafactuales descendentes es mayor, puesto que utilizan el razonamiento contrafactual para compensar y mantener su conducta derrotista ante eventos estresantes de la vida cotidiana, por ejemplo, "Si no me hubiera sacado un 5 no habría aprobado la exposición oral”, lo cual implica "Al menos obtuve un aprobado en la exposición oral”. En estos casos, la inacción y los actos con resultados mediocres se justifican con un eventual resultado contrafactual igual o aún más negativo que los eventos reales (v.g., "Si me hubiera presentado al examen de la primera convocatoria, habría sido igual”), o bien rebajando las expectativas personales respecto a las estándar ("Si no me hubiera presentado al examen, no hubiera aprendido nada, así que da igual que haya suspendido", lo cual implica "suspendí la asignatura, pero al menos sé que aprendí mucho y me conformo con eso"). Los contrafactuales descendentes tienen un doble efecto, por un lado contemplar la posibilidad de haber alcanzado un resultado aún más negativo que el estándar, por ejemplo, ante un mal resultado: "Esto hubiera podido ser peor” y, por otro, la idea de que no había más alternativas, así en "Si hubiera estudiado más, habría suspendido igualmente”, de modo que "no había nada que hacer", que es la perspectiva de las personas con escasa motivación personal (ver Sirois 2004). En general, las personas con baja autoestima tienden a construir más contrafactuales de tipo descendente, mientras que las de alta autoestima tienden a preferir los ascendentes. Esto ocurriría porque los de baja autoestima suelen atribuirse los fracasos como consecuencia de sus propias acciones, de modo que llegan a aceptar los eventos negativos y a imaginarse cómo podrían llegar a ser peores. Por el contrario, los individuos de alta autoestima atribuyen los éxitos a su propia actuación y tienden a pensar en resultados más positivos o de mayor superación personal (Kasimatis y Wells 1995). Las mujeres, ante situaciones de negociación con riesgo social, construyen más contrafactuales ascendentes que los hombres cuando hacen su primera oferta, debido a que a las mujeres les provoca mayor ansiedad el tipo de negociaciones donde están comprometidas las relaciones personales. Esta culpa, expresada generalmente con contrafactuales ascendentes, aumenta cuando las normas para negociar con otro son ambiguas; sin embargo, cuando la ambigüedad respecto a las normas es baja, las mujeres reaccionan igual que los hombres. Los autores concluyen que las reacciones 
emocionales de las mujeres predominan en las negociaciones cuando el contexto no está completamente determinado (Kray y Gelfand 2009).

Todo lo anterior indica que el tipo de contrafactual depende de la personalidad del individuo, pero también de factores externos como la situación particular en la que los hechos ocurren. Así en situaciones en que un individuo sabe de antemano que se enfrentará a una tarea repetitiva, la producción de contrafactuales de tipo ascendente aumentará (Wong et al. 2009). Por otra parte, en casos de catástrofes naturales o accidentes de tráfico, el contrafactual es un recurso para expresar la culpa ante los infortunios de la vida; éste sería "el lado oscuro" del contrafactual. Así hay personas que sufren profundos sentimientos de culpa por haber perdido algún ser querido en un accidente o haber sufrido un aborto involuntario, porque se plantean obsesivamente "qué podrían haber hecho para que las cosas fueran diferentes". Este tipo de contrafactual emplea típicamente la fórmula "si sólo hubiera..." y se dirige a aspectos de la vida inevitables o que no están bajo nuestro control, dejando sin cumplir la función resolutiva del contrafactual, esto es, hacer que las cosas sean mejor. El pensamiento contrafactual más adaptativo se basa en hechos cotidianos, que se repetirán nuevamente, de ahí su función preparatoria para el futuro, pero no en hechos que no volverán a repetirse o cuya probabilidad de ocurrencia es muy baja como la muerte de un ser querido en un terremoto. Por último, los contrafactuales adaptativos son de corta vida, desaparecen tan rápido como aparecen, en cambio, en un estado de depresión podemos pasar toda la vida rumiando contrafactuales de hechos que no volverán a repetirse (Roese 2005). Asimismo, los pacientes con trastorno de la personalidad como la esquizofrenia disminuyen su capacidad para crear contrafactuales de tipo ascendente, debido a que los contrafactuales sirven como reguladores de la conducta social (Wong et al. 2009).

No sólo las diferencias de personalidad determinan el tipo de contrafactual más usado, sino que algunos estudios recientes han encontrado que las diferencias de lateralización manual predicen también tendencias en el uso de contrafactuales. Jasper et al. (2008) en una tarea de producción de contrafactuales ante escenarios negativos y positivos descubrieron que individuos ambidiestros generaron más contrafactuales de tipo ascendente y descendente que los individuos diestros o zurdos. Esto confirma el supuesto de que los participantes ambidiestros pueden mantener múltiples escenarios contradictorios a la vez; en cambio, los individuos con una sola tendencia manual marcada, zurdos o diestros, disminuyen la producción de diferentes tipos de contrafactuales, en este caso, la combinación de contrafactuales de tipo ascendente y descendente. Estas evidencias fisiológicas podrían relacionarse con el hecho de que la activación del hemisferio derecho está asociada a la representación de contenidos contradictorios o incongruentes como es el caso del contrafactual, que aparentemente niega hechos reales, para hacer conjeturas sobre falsas creencias. Nuevos estudios de diferencias individuales en el ámbito de la comprensión lectora con contrafactuales establecen diferencias entre buenos lectores y malos lectores. Principalmente, estas diferencias se encuentran en la detección temprana de las dificultades de integración semántica de un contrafactual en su contexto de discurso. Así los buenos lectores ofrecen un incremento mayor de negatividad en el componente N400 de los potenciales cerebrales (ERP) que los malos lectores cuando leen un contrafactual. Estos últimos, en un estadio más tardío, alrededor de los $600 \mathrm{~ms}$., manifiestan una mayor positividad en el contrafactual. Este último efecto P600 implica procesos de revisión semántica, por tanto, los malos lectores también 
comprenden de manera diferenciada el contrafactual, con un retraso temporal en relación con los lectores más avezados (para más detalle, ver Urrutia et al. 2009).

Los estudios anteriores son, sin duda, muy valiosos para aproximarnos a las diferencias individuales en la producción de contrafactuales. No obstante, con la excepción del último mencionado (Urrutia et al. 2009), se basan en metodologías algo cualitativas, que emplean medidas en escalas de evaluación y que requieren un juicio metacognitivo de los sujetos, en lugar de los métodos conductuales de la psicolingüística o de las neurociencias, que ofrecen medidas más sofisticadas y directas de los procesos (ver Kasimatis y Wells 1995; Roese 2005 y Wong et al. 2009).

\section{CONTRAFACTUAL Y EMOCIÓN}

Como se ha mencionado anteriormente, la comparación entre la realidad y una posibilidad imaginada induce o amplifica emociones contrafactuales, muchas de las cuales son francamente negativas, especialmente aquéllas que, como la culpa o el pesar, se derivan de contrafactuales ascendentes (Kanheman y Tversky 1982). Sorprende este carácter negativo de las emociones contrafactuales, pues podría parecer poco práctico o “adaptativo”. ¿Por qué lamentarse de hechos pasados que no se pueden recuperar? Da la impresión de que el contrafactual cumple una función meramente autopunitiva. Sin embargo, aun cuando los contrafactuales están vinculados a estados emocionales aversivos, cumplen una cierta función hedónica al suavizar un hecho real negativo para fugazmente inducir un estado emocional positivo ante la simulación de buenos resultados, como es el caso de los contrafactuales ascendentes (Roese y Olson 1997).

Las reacciones emocionales positivas de deleite pueden acompañar la recreación de una posibilidad distinta de la que ha obtenido un mal resultado, sobre todo considerando que los resultados negativos son producto de malas decisiones tomadas por nosotros mismos (ver Atkinson et al. 2009). La realización de un acto que podría haber sido mejor puede dar lugar a la esperanza, a una nueva motivación para afrontar acciones futuras de carácter positivo; de ahí que los sentimientos negativos como la culpa o el lamento sirven para mitigar los efectos producidos por los eventos reales, sobre todo cuando se toma una perspectiva de mejora futura. Gracias a la fantasía del contrafactual podemos evadirnos de sentimientos de impotencia y frustración que acompañan a los hechos negativos (Roese y Olson 1995). Todo esto en cuanto al contrafactual ascendente, puesto que el contrafactual descendente se caracteriza por ser una especie de promotor de afectos positivos, conduciendo a sentimientos de orgullo ante determinadas conductas (e.g., "Suspendí la asignatura, pero al menos sé que aprendí mucho"), de gratitud cuando se trata de factores externos controlables (e.g., "El billete me salió caro, pero al menos la azafata fue muy amable conmigo") $\mathrm{y}$, por último, sentimientos de buena suerte cuando los factores son externos, pero volitivos (e.g., "Al menos, la luz eléctrica se fue justo cuando acababa de imprimir el informe"). En definitiva, tanto contrafactual ascendente como descendente cumplen una función hedonista en el estado mental del individuo al mitigar las consecuencias negativas de un resultado (Roese y Olson 1995).

No obstante el argumento anterior, otras investigaciones en el ámbito social (Markman et al. 2009) plantean que los contrafactuales ascendentes generan más costes que beneficios en la vida actual, pues están asociados a sentimiento de 
ansiedad o temor más que a resultados positivos. Al parecer, todo depende del tipo de pensamiento contrafactual; así generar contrafactuales realistas sobre aspectos controlables de un evento negativo (v.g., "Si hubiera dedicado más tiempo, habría aprobado el curso") provoca un efecto diferente que generar contrafactuales sobre aspectos menos viables o incluso irracionales (v.g., "si me hubiera memorizado todos los capítulos, habría aprobado el curso"). El primer ejemplo cumple una función adaptativa al prepararnos para una acción determinada en el futuro, el segundo sólo contribuye a perpetuar un sentimiento negativo al planificar metas que van en contra de acciones constructivas (Markman et al. 2009). En definitiva, los contrafactuales ascendentes, más comunes que los contrafactuales descendentes, no son más que un "reproche mental" que lanzamos constantemente hacia nosotros mismos o hacia los demás cuando la causa es externa. Un reproche mental que sin coste alguno nos previene virtualmente de circunstancias no placenteras en el futuro más cercano.

Otras emociones vinculadas a los contrafactuales ascendentes son las de indignación, pena, envidia, auto-castigo y responsabilidad. En este punto, Kahneman (1995) hace una distinción a partir de la intensidad del contrafactual, sugiriendo una clasificación entre lamento intenso (hot regret) y lamento de tipo melancólico (wistful regret). El primero parece estar relacionado con un arrepentimiento sobre eventos acaecidos recientemente y está acompañado normalmente de rabia e indignación (e.g., "si hubiera llegado cinco minutos antes habría cogido el avión”), mientras que el contrafactual de tipo melancólico responde generalmente a situaciones ocurridas mucho tiempo atrás como una fantasía placentera y triste de lo que podría haber sido, cuyo único límite es la imaginación (e.g., "Si hubiera dedicado más tiempo a mis hijos...”) (Gilovich y Medvec 1994; Kahneman 1995).

\section{TIPOS DE CONTRAFACTUAL}

\subsection{Contrafactuales reales vs fantásticos}

Precisamente a partir de la imaginación sin cortapisas, propia del pensamiento creativo, se puede elaborar contrafactuales fantásticos que alteren arbitrariamente las propiedades ontológicas de ciertas entidades. Por ejemplo: "si tuviera alas, me iría volando a mi oficina todos los días", "si los gatos fueran vegetarianos, le daría de comer lechuga al mío", o bien "si tuviera un ojo en el cuello, no necesitaría retrovisores en el coche". Notoriamente este tipo de contrafactuales no es de uso frecuente. ¿Qué sentido tiene preguntarse si los gatos fueran vegetarianos?. Sin duda, se trata de un tipo de contrafactuales diferentes a los de uso cotidiano, pues requieren una elaboración especulativa de la realidad, que implica una alteración ontológica, o al menos muy improbable, del funcionamiento del mundo. Su uso está casi exclusivamente limitado a elaboraciones literarias como los mitos o la ciencia ficción y su función pragmática en contextos naturales de comunicación es nula a diferencia de las múltiples funciones de los contrafactuales ascendentes y descendentes ya mencionadas. Curiosamente, algunos estudios psicolingüísticos sobre contrafactuales han elegido precisamente este tipo de contenidos fantásticos como material experimental (Ferguson y Sanford 2008). Por el contrario, nuestro enfoque en este trabajo está puesto en contrafactuales de carácter común o cotidiano. 
Los contrafactuales más comunes son simulaciones realistas, propios de una “imaginación racional” (Byrne 2005) y resultan mucho más interesantes desde un punto de vista cognitivo que las fantasías contrafactuales antes mencionadas. Su realismo se basa en que plantean un estado alternativo del mundo que supone una alteración mínima y plausible de los eventos reales (Kahneman y Tversky 1982; Roese 2005 y Byrne 2005). Por ejemplo, se menciona un pequeño cambio temporal (“si hubiera llegado 5 minutos antes, habría cogido el avión”), espacial ("si hubiera estado en la reunión, hubiera votado en contra”), de acción/inacción ("si me hubiera callado habría evitado la crisis”), etc. La principal diferencia entre los contrafactuales comunes y los contrafactuales fantásticos está en el tipo de alternativas utilizado, pues en el primero los hechos reales son presupuestos (e.g., "no llegó 5 minutos...”, "no estaba en la reunión...”, "no me callé la boca...”) mientras que en el segundo las alternativas son inventadas (e.g.,"si a los árboles les crecieran monedas, seríamos millonarios”). Nótese que este tipo de estructuras no obedecen a un estricto contrafactual, pues los eventos se cuentan en un pre-futuro (prefactual), utilizando el verbo en tiempo presente.

Los procesos cognitivos que subyacen a las contrafactuales fantásticos o realistas comparten la misma estrategia mental: guardar en mente dos estatus epistémicos distintos, uno real y otro falso, como si este último fuera verdadero temporalmente. Sin embargo, desde un punto de vista pragmático, los contrafactuales realistas o cotidianos se ajustan mucho mejor a las máximas o principios griceanos de la comunicación (Levinson 1995), especialmente el de calidad (céntrese en lo verdadero) y el de relevancia (vaya directo al grano). Ambos principios, sin duda, contribuyen a que los contrafactuales basados en hechos reales sean más naturales en la comunicación social (Byrne y Girotto 2009).

Además de su uso pragmático en contextos cotidianos, los contrafactuales desempeñan un papel importante en el pensamiento hipotético, fundamental para la reflexión científica. Una práctica normal en ciencias es el uso de la argumentación hipotética en la que se establecen proposiciones contrafactuales para explorar posibles relaciones casuales a modo de “experimentos mentales”. En este contexto de razonamiento científico es donde pueden plantearse contrafactuales "fantásticos" alejados de las restricciones realistas del contrafactual cotidiano. Así por ejemplo, el principio antrópico en la cosmología moderna evalúa las condiciones físicas iniciales de nuestro universo y plantea de modo contrafactual universos alternativos en que las condiciones iniciales hubiesen sido diferentes. Así "si la constante de gravitación hubiera sido mayor, las estrellas se consumirían a mayor velocidad y, posiblemente, nunca hubiera sido posible la existencia de planetas con condiciones adecuadas para la existencia de la vida y por tanto no estaríamos aquí haciéndonos preguntas”. Otros ejemplos de experimentos mentales serían los formulados por Einstein, como se expresa en el siguiente enunciado: "Imagínate que vas en un tren que se desplaza a la velocidad de la luz y contemplas un rayo de luz que camina en paralelo, ¿cómo se vería?”. El experimento de la habitación china de Searle (1980) es un nuevo ejemplo: “Imagínate que estás en una habitación rodeada de manuales de instrucciones y recibes hojas con caracteres chinos del exterior., gracias al manual eres capaz de responder a cualquier texto en chino, aunque nunca hayas hablado o leído ese idioma. ¿Cómo puedes responder si no entiendes el idioma?". 


\subsection{Contrafactuales aditivos y sustractivos}

De acuerdo a su estructura, el contrafactual se ha dividido en dos tipos: aditivo y sustractivo. El primero se relaciona directamente con los contrafactuales ascendentes, dado que se refiere a la suma de nuevos antecedentes para reconstruir la realidad, así en "si me hubiera comprado un portátil, el trabajo lo hubiera hecho mucho mejor". En cambio, los contrafactuales sustractivos, relacionados con los contrafactuales de tipo descendente, van en dirección contraria, pues reducen los elementos del antecedente para reconstruir la realidad. Es el caso de "si no hubiera bebido cerveza la última noche, el trabajo lo habría hecho mucho mejor”, donde sólo la diferencia entre beber o no cerveza cambia el resultado del problema. Los contrafactuales aditivos son de carácter expansivo, facilitando la generación de nuevas ideas y se utilizan frecuentemente en la publicidad y el marketing para crear falsas expectativas en el pensamiento del consumidor al mostrarnos una vida contrafactual en la que tenemos una mejor figura, un marido atento, una casa formidable sólo por comprarnos un coche nuevo. Por su parte, los contrafactuales sustractivos establecen un estilo de procesamiento relacional que beneficia la exactitud en la toma de decisiones y la actuación de tareas de asociación creativa como el insight y asociaciones remotas. De ahí que el pensamiento contrafactual no sólo crea experiencias fenomenológicas para facilitar el pensamiento estructurado como en el caso del razonamiento científico, sino que también modula la creatividad humana gracias a su estilo de procesamiento relacional entre la realidad y lo que podría haber sido, promoviendo el pensamiento analítico, en el caso del contrafactual sustractivo, y la imaginación de carácter estructurada, en el caso del contrafactual aditivo.

En conclusión, los usos y los significados de los contrafactuales son múltiples y diversos. En los debates formales o académicos se utilizan para construir argumentos que justifican nuestras hipótesis para tratar de persuadir al oyente o para modificar sus creencias, hacer prescripciones, advertencias, dar sentido implicativo, refutar los postulados contrarios, formular leyes, normas, contrastar una realidad con otra, etc. Fuera del contexto científico, los actos de habla más típicos del contrafactual son reprochar o recriminar, amenazar, expresar nuestros deseos, sentimientos, temores o esperanzas (García 2009).

\section{LA REPRESENTACIÓN DEL CONTRAFACTUAL: TEORÍAS}

Supongamos que nuestro vecino nos dice: "si hubiera comprado aquel boleto de lotería me habría tocado el premio gordo". Comprender un contrafactual como éste implica la activación de un significado dual. Por una parte hay una doble negación del antecedente y del consecuente, es decir, que mi vecino "no compró el boleto" y "no le tocó el premio gordo; formalmente: "no-p \& no-q". Por otra parte, se simula el estado del mundo alternativo, es decir " $p$ \& q", en el que el antecedente y el consecuente se actualizan mentalmente" como si fueran reales (mi vecino compró el boleto y le tocó el premio gordo). El contrafactual tiene, pues, un doble estatus: una representación realista de los eventos (la doble negación implícita) y otra puramente hipotética o irreal. Una función cognitiva del contrafactual es inducir una toma de perspectiva en el oyente o lector, puesto que automáticamente nos ponemos en el lugar de la persona que podría haber ganado el gordo de la lotería. El contrafactual también nos lleva 
a adoptar una retrospectiva de los sucesos, transportándonos a un espacio temporal en que las cosas pudieron ser diferentes. Perspectiva y retrospectiva implican la inhibición de las propias creencias sobre los hechos reales para dar paso a unas creencias alternativas sobre unos hechos virtuales. Este desdoblamiento cognitivo tiene relación con nuestras capacidades mentalistas o lo que algunos autores han denominado la Teoría de la Mente (Leslie 1987; Leslie et al. 2004).

El significado dual de los contrafactuales ha sido ampliamente estudiado por la psicología social y la psicología del razonamiento (Kahneman y Tversky 1982; Roese 2005; Byrne 2002, 2005; Thompson y Byrne 2002; Johnson-Laird y Byrne 2002 y Santamaría, et al. 2005). El énfasis se ha puesto en el rol de los contrafactuales en el razonamiento causal, en los juicios predictivos, en la toma de decisiones, o en el aprendizaje a partir de los errores (Harris et al. 1996; Wells y Gavanski 1989; Roese y Olson, 1997; Spellman y Mandel 1999 y Mandel 2003). Todos estos estudios analizan el uso del contrafactual desde la perspectiva del hablante o del razonador. Sin embargo, desde el punto de vista de la comprensión, pocos estudios han abordado el análisis de los contrafactuales.

A continuación, revisaremos algunos planteamientos teóricos e investigaciones empíricas relevantes que, aun procediendo del campo del razonamiento, ofrecen datos e ideas importantes para el tema que nos ocupa, es decir, la comprensión de contrafactuales. En primer lugar, mencionaremos dos teorías (la de los espacios mentales y la de los modelos de situación), que mantienen la noción de dualidad representacional ya mencionada. En segundo lugar, trataremos la teoría suposicional, que critica a los modelos mentales, pues considera que los contrafactuales implican una única representación.

\subsection{Espacios mentales}

Una forma de describir el carácter realista de los contrafactuales es mediante la noción teórica de espacios mentales (Fauconnier 1994; Fauconnier y Sweetser 1996). Las claves lingüísticas del contrafactual (condicional si + modo subjuntivo, etc.), funcionan como "constructores de espacios" (space builders) que guían al oyente/lector a establecer dos representaciones: un espacio base correspondiente a los eventos en la situación real y un segundo espacio mental que incluye la situación alternativa. Entre ambos espacios existe un gran paralelismo al compartir entidades como los personajes, los roles, los escenarios y los objetos. No obstante, hay algún parámetro que difiere entre ambos espacios mentales, relacionado, como veíamos, con el espacio, el tiempo, la acción, etc., de modo que ambos espacios son incompatibles entre sí y permanecen compartimentados sin integrarse (Leslie 1987).

La teoría lingüística de los espacios mentales de Fauconnier plantea ideas interesantes. Sin embargo, aporta un análisis formal carente de hipótesis psicológicas verificables. Por esta razón, no se han desarrollado experimentos sobre contrafactuales en el contexto de los espacios mentales. Las siguientes teorías, en cambio, están asociadas a programas de investigación empíricos.

\subsection{Modelos mentales}

La teoría de los modelos mentales se acomoda muy bien al estudio del doble significado de los contrafactuales (Johnson-Laird y Byrne 2002 y Byrne 2002, 
2005). Según sus autores, la comprensión de un contrafactual supone una activación simultánea de dos modelos de situación alternativos ("no-p \& no-q" y "p \& q"), tal como se ilustra en el ejemplo mencionado al comienzo de esta sección. En este sentido, la teoría de los modelos mentales es equivalente a la teoría de los espacios mentales de Fauconnier, mencionada anteriormente, aunque, a diferencia de aquélla, ofrece una gran variedad de experimentos para verificar sus propuestas.

Los estudios sobre contrafactuales, desarrollados en el marco de los modelos mentales, plantean generalmente premisas contrafactuales y solicitan de los participantes que emitan juicios de valores de verdad, eligiendo entre alternativas predeterminadas: p y q; no-p y no-q; p o q. Así, por ejemplo, Thompson y Byrne (2002) pidieron a un grupo de participantes que evaluasen argumentos lógicos a partir de un contexto. Para ello utilizaron frases condicionales en indicativo: "Si Sara fue a Los Ángeles, entonces Rosa fue a Chicago”, o bien en subjuntivo (contrafactuales): "Si Sara hubiera ido a los Ángeles, entonces Rosa habría ido a Chicago", seguida cada una de ellas por la aseveración de un personaje que afirmaba o negaba que "Sara fue a Los Ángeles". La tarea de los sujetos consistía en elegir entre varias opciones la verdad o falsedad de su antecedente o su consecuente en algunos casos. En el ejemplo, "Rosa fue a Chicago", "Puede que Rosa haya ido o no haya ido a Chicago", "Rosa no fue a Chicago", fueron algunas de las opciones. Como se puede comprobar, el tipo de oraciones utilizadas se refiere al uso de tablas de verdad lógicas, propias del área del razonamiento, que distan bastante del uso habitual del condicional en contextos cotidianos.

En otro estudio, utilizando medidas de tiempo de lectura en lugar de selección de respuestas, Santamaría et al. (2005) mostraron a los participantes condicionales contrafactuales ("si hubiera habido rosas entonces habría habido lilas") o bien condicionales factuales ("si hay rosas entonces hay lilas") inmediatamente antes de una determinada oración crítica. Dicha oración crítica podría ser afirmativa ("había rosas y había lilas") o negativa ("no había rosas y no había lilas"). Las oraciones factuales facilitaron la lectura sólo de la oración afirmativa, mientras que las oraciones contrafactuales facilitaban tanto las oraciones afirmativas como negativas, indicando claramente que los lectores generan una doble representación.

\subsection{Teoría suposicional}

Una de las críticas más importantes a la teoría de los modelos mentales y su aplicación a la contrafactualidad la realiza la teoría suposicional (Evans et al. 2005; Evans 2006). Dicha teoría, al contrario que la teoría de los modelos mentales, postula que las proposiciones hipotéticas y particularmente los condicionales, incluidos los contrafactuales, determinan una única representación correspondiente a la suposición planteada literalmente. Por ejemplo en "si hubiera comprado aquel boleto de lotería me habría tocado el premio gordo", el lector/oyente representaría exactamente el sentido literal de la suposición "si p entonces q". Es decir, se representa las probabilidades del consecuente (tocarle a uno el premio gordo) que se derivan de la condición dada (comprar aquel boleto de lotería).

Evans (2006) destaca que, a diferencia de la teoría de los modelos mentales, su modelo es de tipo epistémico, puesto que no sólo representa estados del mundo, sino también las intenciones y creencias que se tengan sobre la realidad. En otras 
palabras, mientras que los modelos mentales sólo representan estados posibles del mundo, la teoría suposicional de Evans refleja también las actitudes proposicionales (hipótesis o suposiciones) sobre estos mismos estados del mundo. Los modelos mentales epistémicos se recuperan directamente de la memoria semántica mediante mecanismos heurísticos, los cuales implican la representación selectiva de contenidos complejos. Sin embargo, Evans plantea que las suposiciones sufren un proceso de desdoblamiento de la memoria semántica para que puedan distinguirse de las creencias reales acerca del mundo. Uno de sus principios es el de la singularidad; de acuerdo con éste, sólo se representaría el contenido hipotético, la probabilidad de que algo ocurra dado un determinado supuesto, más que lo que en realidad no ocurrió. En este sentido, el enfoque suposicional de Evans es, según él mismo declara, mucho más pragmático que las teorías competidoras, pues se acomoda al principio de relevancia, de acuerdo al cual sólo se representaría la posibilidad más cercana, la que menos cambios supone al mundo real, esto es, la creencia falsa de la realidad.

Aunque la inclusión de la inferencia pragmática es un aspecto interesante de la teoría suposicional, hay relativamente pocos estudios experimentales que hayan verificado los supuestos básicos de relevancia y activación de una sola representación. La mayoría de los estudios, como los que hemos revisado en el apartado anterior, concluyen que se activan dos modelos mentales en la comprensión de un contrafactual (Johnson-Laird y Byrne 2002; Byrne 2002, 2005 y Santamaría et al. 2005). No obstante, en un trabajo reciente se intentó aportar datos a favor de la teoría suposicional (Stewart et al. 2009). En dicha investigación, que describiremos en detalle en la próxima sección, los autores, mediante una tarea de lectura autoespaciada palabra por palabra, hallaron evidencia favorable a la teoría suposicional sólo cuando los participantes leían condicionales indicativos ("Si Darren es atlético, probablemente jugará en el equipo de rugby"), pero no en los condicionales contrafactuales ("Si Darren hubiera sido atlético, probablemente habría jugado en el equipo de rugby”), por tanto los resultados falsean más que confirman la teoría de Evans.

Los estudios de modelos de situación y del enfoque suposicional tratan de valorar ciertas teorías del razonamiento lógico, sin ajustarse al uso pragmático de los contrafactuales que tan bien han descrito los psicólogos sociales. Cuando escuchamos o leemos un contrafactual, al menos en contextos pragmáticos naturales, probablemente la tarea primaria en muchos casos no es establecer la probabilidad condicional de los eventos contrafactuales o derivar las consecuencias un silogismo condicionas, sino únicamente la comprensión de los enunciados contrafactuales, sin tareas lógicas adicionales.

\section{COMPRENSIÓN DE CONTRAFACTUALES}

Dada la probada funcionalidad psicológica de los contrafactuales y su ubicuidad en contextos narrativos, conversacionales y de razonamiento cotidiano y científico, es notable la relativa ausencia de estudios psicolingüísticos y de neurociencia cognitiva sobre la comprensión de estos enunciados. Sólo recientemente se han desarrollado algunas investigaciones sobre el curso temporal de la comprensión de oraciones contrafactuales (de Vega et al. 2007; Ferguson y Sanford 2008 y Stewart et al. 2009). A diferencia de los anteriores experimentos, en éstos se compararon únicamente 
oraciones factuales frente a oraciones contrafactuales. En el trabajo de de Vega et al. (2007), los lectores debían leer breves historias que comenzaban con la descripción de una situación factual ("Juan estaba en la oficina, sentado en frente del ordenador. Comenzó a teclear un informe que le había pedido su jefe”). Después de esta situación inicial, la frase crítica podía ser factual o contrafactual ("Como Juan tenía bastante tiempo fue al bar a beber una cerveza" vs. "Si Juan hubiera tenido bastante tiempo habría ido al bar a beber una cerveza"), para finalizar con una oración de relleno de carácter neutro. Se utilizó una medida de activación, esto es, una palabra de prueba perteneciente al contexto inicial, en este caso el verbo "teclear”, y otro verbo perteneciente a la cláusula subordinada en el ejemplo "beber”. Nótese que ambas palabras de prueba estaban presentes en los dos contextos: factual y contrafactual y en el mismo contexto local, por tanto, las diferencias léxicas fueron controladas.

Los resultados indicaron que los lectores fueron más lentos al identificar la palabra de prueba inicial (teclear) en el factual que en el contrafactual, resultados que apoyan la idea de actualización del modelo de situación en el caso del factual y cancelación del mismo en el contrafactual. El resultado de la palabra 2 (beber), en cambio, fue más complejo; las respuestas fueron igualmente rápidas en el contrafactual que en el factual cuando dicha palabra fue presentada inmediatamente después de leer la frase crítica, mientras que, después de la frase de relleno, las respuestas fueron más rápidas en la versión factual que en el contrafactual. Estos resultados sugieren que en los contrafactuales no sólo se mantienen activados los eventos vinculados al modelo de situación inicial, sino que la información nueva también llega a representarse momentáneamente, dada su dualidad representacional (no-p y no-q; p y q).

Ferguson y Sanford (2008), por su parte, utilizando un tipo de contrafactual de carácter artificioso ("Si los gatos fueran vegetarianos, sería más barato para sus dueños cuidar de ellos") y un condicional factual ("Si los gatos estuvieran hambrientos, normalmente molestarían a sus dueños hasta lograr comida”), realizaron la misma manipulación del estudio anterior, sólo que esta vez emplearon la técnica de registro de movimientos oculares. Sus resultados indican que inmediatamente después de leer una oración condicional factual, la lectura de una oración consistente con el mundo real ("las familias podrían alimentar a sus gatos con un bol de pescado...") fue más rápida que la lectura de frases consistentes con el mundo contrafactual ("las familias podrían alimentar a su gato con un bol de zanahorias”). Sin embargo, en el caso de un contexto contrafactual ocurrió exactamente lo mismo, puesto que las oraciones consistentes con el mundo contrafactual fueron más lentas que las oraciones inconsistentes. Sólo en un momento posterior, la lectura de la oración consistente con el contexto contrafactual fue más rápida. Los resultados sostienen la idea de que dos posibilidades (no-p y no-q; p y q) están vinculadas al contexto contrafactual, mientras que una sola lo está al contexto factual, tal cual plantea la teoría de los modelos mentales.

Otro estudio de los mismos autores (Ferguson et al. 2008), utilizando la técnica de ERP, demostró que las palabras críticas consistentes con el escenario contrafactual provocaron una N400 mayor que las palabras críticas coherentes con el mundo real. Estos resultados (experimento 2) confirman la idea de que los lectores no pueden suprimir inicialmente su conocimiento del mundo a pesar de la presuposición contrafactual, que en este caso es mucho más latente al violar ese conocimiento. Sin embargo, esta negatividad se invirtió más tarde en otras palabras de la misma 
oración crítica, de modo que se observó el patrón habitual de incremento en N400 para palabras inconsistentes con el contrafactual. Los resultados sugieren que en una segunda etapa, los lectores pudieron resolver el conflicto entre el mundo real y la información proveniente del contrafactual.

Finalmente, en el ya mencionado estudio de Stewart et al. (2009), se trató de verificar la teoría suposicional de Evans. La idea de este estudio (experimento 2) era contrastar condicionales de indicativo y condicionales de subjuntivo (contrafacuales) con una presuposición dada de antemano en la que se presentaba un contexto donde se negaban los hechos ("Darren no era atlético en absoluto") y otro en el que se afirmaban ("Darren era muy atlético"). El objetivo era saber si la información condicional se representaría inicialmente en una etapa temprana sin hacer referencia al modelo de situación del mundo real, tal como predice el modelo suposicional de Evans et al. (2005) y Evans (2006). Los resultados mostraron que los participantes leían más rápido sólo la palabra final del antecedente del contrafactual (e.g., "atlético") y la primera del consecuente ("habría jugado"), cuando la información era consistente con el contexto ("Darren no era atlético en absoluto"), que cuando era inconsistente ("Darren era muy atlético"). Los resultados no confirman las predicciones de la teoría suposicional, puesto que no se observó tiempos de lectura menores entre el antecedente contrafactual y un contexto previo relacionado con la representación alternativa ("Darren era atlético"), presente en la representación dual del contrafactual. En cambio, los condicionales indicativos no mostraron diferencias temporales entre el contexto previo incoherente con la cláusula inicial condicional, lo cual indica que los condicionales de tipo indicativo no se vieron afectados por el contexto previo. Esto último apoyaría la teoría suposicional de Evans, la cual plantea que la información proveniente de los condicionales se mantiene aparte, segregada de lo que el lector conoce de la situación real (ver Stewart et al. 2009).

En suma, la teoría de los espacios mentales (Fauconnier 1994, Fauconnier y Sweetser 1996), la teoría de los modelos mentales y las diversas investigaciones provenientes de la psicología social y del razonamiento dan a entender la existencia de que la semántica del contrafactual implica una doble representación. Los estudios sobre razonamiento contrafactual, por su parte, se centran en la idea de que el lector representa preferentemente información verdadera, pues el lector tiene un modelo mental de la situación real (no-p \& no-q), sin embargo, a través de las inferencias imaginarias, el lector es capaz también de construir un modelo mental de la situación irreal ( $p$ \& q) en el caso del contrafactual. Los estudios empíricos detallados en sesiones anteriores dan respaldo a la teoría de los modelos mentales (Byrne y Tasso, 1999; Johnson-Laird y Byrne 2002; Byrne 2002; Santamaría et al. 2005).

Por el contrario, la propuesta de Evans sobre el carácter suposicional y, por consiguiente, de representación única del contrafactual, no se confirma, puesto que Steward et al. (2009) sólo encontraron un respaldo a esta teoría en los condicionales de indicativo, pero no en los contrafactuales. Por su parte, Ferguson et al. (2008) confirman esta hipótesis parcialmente al encontrar una interferencia temprana en oraciones que son incoherentes con el mundo real. Así, estos autores encontraron inferencias más rápidas en oraciones que presentaban un cambio mínimo de la realidad, por ejemplo "gatos comiendo zanahorias" ante un contexto previo como "los gatos son vegetarianos". Esto significa que cuando el contexto corresponde a un significado contrafactual, la información congruente con el mundo real ("los 
gatos son carnívoros") se procesa como un significado anómalo. No obstante lo anterior, la información contextual acerca de la realidad influye en el procesamiento contrafactual en una fase tardía, de modo que estos resultados, en definitiva, también apuestan por un procesamiento dual del contrafactual (Ferguson et al. 2008).

\section{CONCLUSIONES}

Comprender un contrafactual supone, entonces, la representación de dos tipos de significados. Una información relacionada con la realidad de los conceptos negados mediante el contrafactual y otra información de carácter perceptual, basada en la información experiencial que se tiene sobre los eventos alternativos a través del contrafactual. Comprender un texto contrafactual implica también la realización de múltiples tareas, el despliegue de más de un escenario, la selección de uno de ellos, la simulación de un estado irreal como si fuera real, la inhibición de una falsa creencia, la toma de perspectiva, la atribución de estados mentales y emocionales, entre otras. Los contrafactuales se distinguen por su estatus de irrealidad, al referirse a hechos que nunca ocurrieron ni ocurrirán. Los pensamientos contrafactuales son inconscientes y automáticos, normalmente vienen a la mente y desaparecen sin ser realizados, pero esto no significa que su uso sea gratuito, sin coste cognitivo (Roese 2005). Por el contrario, el contrafactual se destaca por su alto grado de mentalización y la posible decodificación de intenciones, relativos probablemente a los procesos derivados de la teoría de la mente, puesto que, tanto en el contrafactual como en las tareas de teoría de la mente debe inhibirse una intención y seleccionar otra a través del sistema ejecutivo central, funciones características del lóbulo prefrontal (Gusnard et al. 2001 y Haynes et al. 2007).

Existen muy pocas evidencias empíricas de neuroimagen directamente relacionadas con la comprensión del lenguaje contrafactual hasta el momento. Sin embargo, en un estudio llevado a cabo recientemente con la técnica de Resonancia Magnética funcional (fMRI) (Urrutia et al. 2010), se compararon oraciones contrafactuales de acción manual ("Si Daniel hubiera celebrado su boda, él habría levantado su copa”) con oraciones factuales de acción manual ("Como Daniel estaba celebrando su boda, él está levantando su copa”). Los resultados indicaron una activación exclusiva para los contrafactuales en áreas prefrontales tales como el cingulado anterior derecho, el giro frontal medial izquierdo y el giro frontal superior derecho. Estas zonas corresponden a las bases neurales del significado dual del contrafactual, comprometidas en las tareas de inhibición de la representación alternativa en la compleja semántica del contrafactual.

\section{OBRAS CITADAS}

Atkinson, L., Bell, D. Y Feeney, A. 2009. "The relationship between counterfactual thinking and emotional reactions to event outcomes: Does one account fit all?" Psychonomic Bulletin \& Review 16, 4: 724-728.

Byrne, R. 2002. "Mental models and counterfactual thoughts about what might have been". Trends in Cognitive Sciences 6, 10: 426-431. 
Byrne, R. 2005. The rational imagination. How people create alternatives to reality. Cambridge, M. A.: MIT Press.

Byrne, R. y Girotto, V. 2009. "Cognitive processes in counterfactual thinking”. D.M., Keith, W.M., Klein, \& J.A. Suhr (Eds.), Handbook of imagination and mental simulation. New York: Psychology Press. 151-160.

Byrne, R. y Tasso, A. 1999. "Deductive reasoning with factual, possible, and counterfactual conditionals”. Memory \& Cognition 27: 309-323.

De Vega, M., Urrutia, M. y Riffo, B. 2007. "Cancelling updating in the comprehension of counterfactuals embedded in narratives”. Memory \& Cognition 35, 6: 1410-1421.

Evans, J. 2006. "The heuristic analytic theory of reasoning: Extension and evaluation". Psychonomic Bulletin \& Review 13 3: 378-395.

Evans, J., Over, D. y Handley, S. 2005. "Supositions, Extensionality, and Conditionals: A Critique of the mental model theory of Johnson-Laird and Byrne (2002)". Psychological Review 112, 4:1040-1052.

Fauconnier, G. 1994. Mental spaces. Aspects of meaning construction in natural language. New York: Cambridge University Press.

Fauconnier, G., y Sweetser, E 1996. Spaces worlds and grammar. Chicago: The University of Chicago Press.

Ferguson, H.J. y Sanford , A.J. 2008. "Anomalies in real and counterfactual worlds: An eyemovement investigation”. Journal of Memory and Language 58: 609-626.

Ferguson, H.J. Sanford, A.J. y Leuthold, H. 2008. "Eye-movements and ERPs reveal the time course of processing negation and remitting counterfactual worlds”. Brain Research 1236: 113-125.

García, J. 2009. "Condición e hipótesis. Más allá de lo real y lo irreal”. Revista de Lingüística y Lenguas Aplicadas 4: 79-88.

Gilovich, T. y Medvec, V. 1994. "The temporal pattern to the experience of regret". Journal of Personality and Social Psychology 67: 357-365.

Gusnard, D. A., Akbudak, E., Shulman, G. L., y Raichle, M. E. 2001. "Medial prefrontal cortex and self-referential mental activity: relation to a default mode of brain function". Proceedings of the National Academic of the Sciences 98: 4259-4264.

Harris, P.L., German, T.P. y Mills, M. 1996. "Children's use of counterfactual thinking in causal reasoning”. Cognition 61: 223-259.

Haynes, J-D, Sakai, K., Rees, G., Gilbert, S., Frith, C. y Passingham, D. 2007. "Reading hidden intentions in the human brain”. Current Biology 17: 323-328.

Jasper, J., Barry, K. y Christman, S. 2008. "Individual differences in counterfactual production". Personality and individual differences 45; 488-492.

Johnson-Laird, P.N. y Byrne, R.M.J. 2002 "Conditionals: a theory of meaning, pragmatics, and inference”. Psychological Review 109: 646-678.

Kahneman, D. y Tversky, A. 1982. "The simulation heuristic". D. Kahneman, P. Slovic y A. Tversky (Eds.) Judgement under uncertainty: Heuristics and biases. New York: Cambridge University Press. 201-208.

Kahneman, D. 1995 "Varieties of Counterfactual Thinking". En N.Roese, \& J. Olson (Eds.). What might have been. The social psychology of counterfactual thinking. Mahwah, New Jersey: Lawrence erlbaum associates, publishers. 375-396.

Kasimatis, M. y Wells, G. 1995. "Individual differences in counterfactual thinking". En N. Roese y J. Olson (Eds.). What might have been. The social psychology of counterfactual thinking. Mahwah, New Jersey: Lawrence Erlbaum Associates, Publishers. 81-101.

Kray, L.J., y Gelfand, M. 2009. "Relief Versus Regret: The Effect of Gender and Negotiating Norm Ambiguity on Reactions to Having One's First Offer Accepted". Social Cognition 27, 3: 418-436.

Leslie, A. 1987. "Pretense and Representation: The origins of "theory of mind”". Psychological 
Review 94, 4: 412-426.

Leslie, A., Friedman, O. y German, T. 2004. "Core mechanisms in "theory of mind"'. Trends in Cognitive Sciences 8, 12: 528-533.

Macmullen, M., Markman, K. y Gavanski, I. 1995. "Living in neither the best nor worst of all possible worlds: Antecedents and consequences of upward and downward counterfactual thinking”. En N. Roese y J. Olson (Eds.), What might have been. The social psychology of counterfactual thinking. Mahwah, New Jersey: Lawrence Erlbaum. pp. 133-167.

Mandel, D.R. 2003. "Effect of counterfactual and factual thinking on causal judgements". Thinking and Reasoning 9, 3: 245-265.

Markman, K.D., Karadogan, F., Lindberg, M.J. y ZELL, E. 2009. "Counterfactual thinking: Function and dysfunction". En D.M., Keith, W.M., Klein, \&, J.A., Suhr (Eds.), Handbook of imagination and mental simulation. New York: Psychology Press. pp.175-193.

Roese, $\mathrm{N}$ y Olson, J. 1995. What might have been. The social psychology of counterfactual thinking. Mahwah, New Jersey: Lawrence Erlbaum Associates.

Roese, N y Olson, J. 1997. "Counterfactual thinking: The intersection of affect and function". En, M. P. Zanna. (Ed.), Advances in experimental social psychology. San Diego, CA: Academic Press.

Roese, N. J., Sanna, L. J. y Galinsky, A. D. 2005. "The mechanics of imagination: Automaticity and control in counterfactual thinking". En J. A. Bargh, J. Uleman, y R. Hassin. (Eds.), The New Unconscious. New York, NY: Oxford University Press. pp. 138-170.

Roese, N. 2005. If only: How to turn regret into opportunity. New York: Broadway Books.

Santamaría, C., Espino, O. y Byrne, R. 2005. "Counterfactual and semifactual conditionals prime alternative possibilities”. Journal of Experimental Psychology: Learning, Memory and Cognition 31: 1149 - 1154.

Searle, J. 1980. "Minds, brains and programs". Behavioral and brain sciences 3, 3: 417-457.

Sirois, R. 2004. "Procrastination and counterfactual thinking: Avoiding what might have been". British Journal of Social Psychology 43: 269-286.

Spellman, B. y Mandel, D. 1999. "When possibility informs reality: Counterfactual thinking as a cue to causality”. Current Directions In Psychological Sciences 8,4: 120-123.

Stewart, A. J., Haigh, M. y Kidd, E. 2009. "An investigation into the en-linea processing of counterfactual and indicative conditionals”. Quarterly Journal of Experimental Psychology 62: 2113-2125.

Thompson, V. y Byrne, R. 2002. "Reasoning Counterfactually: Making inferences about things that didn't happen". Journal of Experimental Psychology: Learning, Memory, and Cognition 28, 6: 1154-1170.

Urrutia, M., Marrero, H., Castillo, M.D., Hernández, J.A., y De Vega, M. 2009. “Individual differences in the comprehension of counterfactual stories. An ERP study". Póster presentado en Nineteenth Annual Meeting of the Society for Text and Discourse, Rotterdam, Netherlands.

Urrutia, M., Gennari, S. y De Vega, M. 2010. "Counterfactuals in action. An fMRI study". Póster presentado en el Congreso Embodied Mind: Perspectives and Limitations. Nijmegen, Netherlands.

Wells, G. L. y Gavanski, I. 1989. "Mental simulation of causality”. Journal of Personality and Social Psychology 53: 421-430.

West, W.C. y Holcomb, P.J. 2000. "Imaginal, semantic, and surface-level. Processing of concrete and abstract words: An electrophysiological Investigation”. Journal of Cognitive Neuroscience 12: 1024-1037.

Wong, E.M., Galinsky, A.D., y Kray, L.J. 2009. "The counterfactual mind-set: A decade of research". En D.M. Keith, W.M. Klein, J.A. Suhr, (Eds.), Handbook of imagination and mental simulation (pp.161-174). New York: Psychology press. 